\title{
Simple Multicloud Models for the Diurnal Cycle of Tropical Precipitation. Part II: The Continental Regime
}

\author{
YEVGENIY FRENKEL \\ Courant Institute for Mathematical Sciences, New York University, New York, New York \\ BOUALEM KHOUIDER \\ Department of Mathematics and Statistics, University of Victoria, Victoria, British Columbia, Canada \\ ANDREW J. MAJDA \\ Department of Mathematics, and Center for Atmosphere Ocean Science, Courant Institute of \\ Mathematical Sciences, New York University, New York, New York
}

(Manuscript received 6 July 2010, in final form 22 December 2010)

\begin{abstract}
The variation of precipitation over land due to the diurnal cycle of solar heating is examined here in the context of a simple multicloud model for tropical convection with bulk atmospheric boundary layer (ABL) dynamics. The model utilizes three cloud types (congestus, deep, and stratiform) that are believed to characterize organized tropical convection based on the first two baroclinic modes of vertical structure in the free troposphere, coupled to the ABL through full bulk boundary layer (FBBL) dynamics, that allow a careful separation between sensible and latent heat surface fluxes. In a land parameter regime, characterized by a strong inversion profile, a large Bowen ratio of 0.4 , and active mixing of sensible heat due to cumulus entrainment and downdraft fluxes at the top of the ABL, the model supports a stable 1-day periodic solution that is characterized by a pronounced $\left(7 \mathrm{~K} \mathrm{day}^{-1}\right)$ afternoon peak in precipitation consistent with observations of tropical precipitation over continental regions. The current study suggests a division of the diurnal cycle of precipitation over land into a cycle of five phases: 1) an overnight phase of a radiative-convective equilibrium (RCE) state between 2000 and 0600 LST; 2) an early morning CAPE buildup accompanied by a sudden rise in precipitation that quickly dries the middle troposphere occurs between 0600 and roughly 1000 LST; 3) a moistening phase between roughly 1000 and 1600 LST; 4) a phase of maximum precipitation between 1600 and 1800 LST that dries the middle troposphere and quickly consumes CAPE; and 5) a rapid remoistening phase that restores the moisture level to sustain the overnight RCE precipitation and connects to phase 1 in the cycle. Sensitivity tests in the model confirm that the late afternoon precipitation maximum over land depends crucially on a strong inversion, the large Bowen ratio, and the active mixing of sensible heat due to cumulus entrainment and downdraft fluxes at the top of the ABL.
\end{abstract}

\section{Introduction}

As discussed in Part I (Frenkel et al. 2011, hereafter Part I), the diurnal variation of tropical precipitation involves three different regimes of behavior depending on geographic location (Kikuchi and Wang 2008): an oceanic regime characterized by an early morning precipitation maximum of moderate strength, a continental regime

Corresponding author address: Boualem Khouider, Department of Mathematics and Statistics, University of Victoria, 3800 Finnerty Road, Victoria BC V8W 3P4, Canada.

E-mail: khouider@uvic.ca characterized by a strong precipitation peak occurring in the afternoon, and a more complex coastal regime that involves phase propagation of the precipitation maximum toward land and/or toward the deep ocean basin depending on the time of the day. We are interested here in modeling the main physical mechanisms that drive the two different regimes of diurnal cycle of tropical precipitation over the ocean and over land, respectively, which remain poorly understood and which present a challenge for current general circulation models (Randall et al. 1991; Yang and Slingo 2001; Dai and Trenberth 2004; Tian et al. 2004; Yang and Smith 2006; Kikuchi and Wang 2008). While Part I focused on the simplest model 
for the oceanic regime, here the diurnal cycle over land is the main emphasis. The strategy here consists of using the simple multicloud model for organized tropical convection (Khouider and Majda 2006, 2008b, hereafter respectively KM06 and KM08) with more elaborate boundary layer dynamics, which among other things allows for a separate treatment of sensible and latent heat surface fluxes and explicit representation of shallow cumulus clouds. Such a model was introduced and studied by Waite and Khouider (Waite and Khouider 2009, hereafter WK09) without the effects of the diurnal cycle.

The multicloud models are complex enough to capture the most of the subtle interactions between different clouds in the tropics and therefore provide the first prototype cumulus parameterization capable of representing multiscale organized tropical convective systems including convectively coupled waves (KM06; KM08; Khouider and Majda 2007, 2008a) and the MaddenJulian oscillation (Khouider et al. 2011). However, these parameterizations are simple enough to be able to track down and understand the physical mechanisms at work in the model. Here we take advantage of this feature to understand the physical mechanisms that drive the diurnal cycle of tropical precipitation over land. As in Part I, the main idea is to look for stable one-day periodic solutions that exhibit features similar to those in observations when the model is forced by a surface (latent and sensible) heating that mimics the diurnal cycle of solar heating, under parameter regimes that characterize the tropical climatology over land. The technical procedure and methodology together with the ocean parameter regime are reported in Part I, whereas the land regime is presented here. Because of the importance of sensible heat fluxes for the diurnal cycle of precipitation over land, only the version of the multicloud model with full bulk boundary layer dynamics is considered here. This is unlike the ocean case considered in Part I where the original multicloud model, for which the boundary layer dynamics are reduced to a single equation for the equivalent potential temperature, is sufficient to explain the diurnal cycle due to the small Bowen ratio there. Nevertheless, it was established in Part I that the model with full boundary layer dynamics (WK09) gave essentially the same mechanism for the diurnal cycle over the ocean as the simpler model (KM08).

The present paper is organized as follows. In section 2, we briefly review the Waite-Khouider (WK) model, which was discussed in some detail in Part I. Here the review emphasizes the diurnal variations of sensible and latent heat surface fluxes and the use of a strong temperature inversion above the atmospheric boundary layer (ABL) that are important for the land regime. The mathematical methodology used here to search for one-day periodic solutions is the same as described in the appendix of
Part I. In section 3, we present the main results, for the land case, consisting of a one-day periodic stable solution characterized by a strong afternoon precipitation peak consistent with observations, and we discuss the physical mechanisms underlying this solution. In section 4 , we present sensitivity tests to highlight the physical parameters that control the diurnal cycle of precipitation over land. Finally, a concluding summary and discussion is given in section 5 .

\section{Formulation of WK model with strong inversion}

\section{a. Governing equations}

Two versions of the multicloud model (KM06; KM08) were presented and used in Part I to simulate the diurnal cycle of precipitation over the tropical oceans, one with the boundary layer dynamics reduced to a single equation for the equivalent potential temperature (averaged over the ABL depth) and one that uses a full bulk boundary layer model following Stevens (2006) that, among other important details, separates latent and sensible heat surface fluxes and has an explicit representation of shallow cumulus clouds, namely the WK model (WK09). Because of the importance of sensible heat fluxes for the diurnal cycle over land, here we consider only the WK model.

We recall that the multicloud models (KM06; KM08; WK09) assume three heating profiles associated with the three main cloud types that are observed to characterize organized tropical convective systems (Johnson et al. 1999): cumulus congestus clouds with heating below and cooling aloft, deep convective towers that heat the whole tropospheric depth, and lagging-stratiform anvils that heat the upper troposphere and cool the lower troposphere. Their dynamical core consists of the momentum and potential temperature equations for the first and second baroclinic modes of vertical structure coupled to the boundary layer (KM06; KM08; WK09). The multicloud models carry an equation for the vertically averaged moisture and use a nonlinear switch function to make systematic transitions between dry tropospheric regimes where deep convection is inhibited while congestus clouds are promoted and moist preconditioned states allow for deep convection.

Although the WK model was presented and discussed in some detail in Part I, the model equations are reported in Table 1 and the associated constants and parameters are reported in Table 2, for the sake of completeness. All equations in Table 1 are given in nondimensional form where the speed of the first baroclinic Kelvin waves, $c_{r} \approx$ $50 \mathrm{~m} \mathrm{~s}^{-1}$, is the velocity scale; the equatorial Rossby radius of deformation, $L_{e} \approx 1500 \mathrm{~km}$, is the length scale; $T=L_{e} / c_{r} \approx 8.33 \mathrm{~h}$ is the time scale; and $\bar{\alpha}=$ $H_{T} N^{2} \theta_{0} / \pi g \approx 15 \mathrm{~K}$ is the temperature unit scale. It is 
TABLE 1. Prognostic and diagnostic equations. See text and Table 2 for details.

\begin{tabular}{|c|c|}
\hline Name & WK model \\
\hline Momentum, zeroth mode & $\frac{\partial \bar{u}}{\partial t}=-\partial_{x} p_{0}-\partial_{x} \bar{u}\left(2 \bar{u}-u_{b}\right)+\frac{E_{u}}{H_{T}} \Delta_{t} u$ \\
\hline Momentum, $j$ th mode, $j=1,2$ & $\frac{\partial u_{j}}{\partial t}-\partial_{x} \theta_{j}+\partial_{x}\left(\bar{u} u_{j}\right)=\frac{\sqrt{2}}{\tau_{T}} \delta_{b} \Delta_{t} u-\frac{1}{\tau_{R}} u_{j}$ \\
\hline Potential temperature, first mode & $\frac{\partial \theta_{1}}{\partial t}-\partial_{x} u_{1}+\bar{u} \partial_{x} \theta_{1}+\sqrt{2} \partial_{x} \bar{u}=H_{d}+\xi_{s} H_{s}+\xi_{c} H_{c}+S_{1}+\frac{\pi}{2 \sqrt{2}}\left[\alpha_{E} \frac{E}{H_{T}} \Delta_{t} \theta+\beta_{D}\left(\frac{M_{d}}{H_{T}}+\partial_{x} \bar{u}\right) \Delta_{m} \theta\right]$ \\
\hline Potential temperature, second mode & $\frac{\partial \theta_{2}}{\partial t}-\frac{1}{4} \partial_{x} u_{1}+\bar{u} \partial_{x} \theta_{2}+\frac{\sqrt{ } 2}{4} \partial_{x} \bar{u}=H_{c}-H_{s}+S_{2}$ \\
\hline Radiative cooling & $S_{i}=-Q_{R, i}^{0}-\tau_{D}^{-1} \theta_{i}$ \\
\hline Free tropospheric moisture & $\begin{array}{l}\frac{\partial q}{\partial t}+\bar{u} \partial_{x} q+\partial_{x}\left[\left(u_{1}+\delta u_{2}\right) q+\left(u_{1}+\tilde{\lambda} u_{2}\right) \tilde{Q}-\bar{u} \tilde{Q}_{0}\right]=-\frac{2 \sqrt{2}}{\pi}\left(H_{d}+\xi_{s} H_{s}+\xi_{c} H_{c}\right) \\
\quad+\frac{E}{H_{T}} \Delta_{t} q+\left(\frac{M_{d}}{H_{T}}+\partial_{x} \bar{u}\right) \Delta_{m} q+\left(1-\alpha_{E}\right) \frac{E}{H_{T}} \Delta_{t} \theta+\left(1-\beta_{D}\right)\left(\frac{M_{d}}{H_{T}}+\partial_{x} \bar{u}\right) \Delta_{m} \theta\end{array}$ \\
\hline Boundary layer potential temperature & $\frac{\partial \theta_{b}}{\partial t}+u_{b} \partial_{x} \theta_{b}=-\frac{E}{h_{b}} \Delta_{t} \theta-\frac{M_{d}}{h_{b}} \Delta_{m} \theta+\frac{1}{\tau_{e}} \Delta_{s} \theta-Q_{R b}$ \\
\hline Boundary layer moisture & $\frac{\partial q_{b}}{\partial t}+u_{b} \partial_{x} q_{b}=-\frac{E}{h_{b}} \Delta_{t} q-\frac{M_{d}}{h_{b}} \Delta_{m} q+\frac{1}{\tau_{e}} \Delta_{s} q$ \\
\hline Boundary layer velocity & $\frac{\partial u_{b}}{\partial t}+\bar{u} \partial_{x} \bar{u}=-\partial_{x} p_{0}-\partial_{x} \bar{u}\left(\bar{u}-u_{b}\right)+\frac{E_{u}}{H_{T}} \Delta_{t} u$ \\
\hline Convective downdraft & $D_{c}=m_{0}\left[1+\mu\left(H_{s}-H_{c}\right) / Q_{R, 1}^{0}\right]^{+} \Delta_{m} \theta_{e}$ \\
\hline Total downdraft/updraft at top of ABL & $M_{d}=\left(D_{c}+h_{b} \partial_{x} u_{b}\right)^{+} / M_{u}=D_{c} / \alpha_{m}$ \\
\hline Scalar entrainment velocity & $E=\left(M_{u}-M_{d}+h_{b} \partial_{x} u_{b}\right)^{+}$ \\
\hline Momentum entrainment velocity & $E_{u}=\left(\frac{h_{b}}{\tau_{T}}+h_{b} \partial_{x} u_{b}\right)^{+}$ \\
\hline Stratiform heating & $\frac{\partial H_{s}}{\partial t}=\frac{1}{\tau_{s}}\left(\alpha_{s} H_{d}-H_{s}\right)$ \\
\hline Congestus heating & $\frac{\partial H_{c}}{\partial t}=\frac{1}{\tau_{c}}\left(\alpha_{c} \Lambda Q_{c}^{+}-H_{c}\right)$ \\
\hline Deep convection & $H_{d}=(1-\Lambda) Q_{d}^{+}$ \\
\hline $\begin{array}{l}\text { Maximum energy available for } \\
\text { deep convection }\end{array}$ & $Q_{d}=\bar{Q}+\tau_{\text {conv }}^{-1}\left[a_{1} \theta_{e b}+a_{2} q-a_{0}\left(\theta_{1}+\gamma_{2} \theta_{2}\right)\right]^{+}$ \\
\hline $\begin{array}{l}\text { Maximum energy available for } \\
\text { congestus convection }\end{array}$ & $Q_{c}=\bar{Q}+\tau_{\mathrm{conv}}^{-1}\left[\theta_{e b}-a_{0}^{\prime}\left(\theta_{1}+\gamma_{2}^{\prime} \theta_{2}\right)\right]^{+}$ \\
\hline $\begin{array}{l}\text { Jump between the surface and } \\
\text { bulk ABL value of } \phi\end{array}$ & $\Delta_{s} \phi=\phi_{s}-\phi_{b}$ \\
\hline $\begin{array}{l}\text { Jump between bulk ABL and top } \\
\text { of ABL value of } \phi\end{array}$ & $\Delta_{t} \phi=\phi_{b}-\phi_{t}$ \\
\hline $\begin{array}{l}\text { Jump between bulk ABL and middle } \\
\text { troposphere value of } \phi\end{array}$ & $\Delta_{m} \phi=\phi_{b}-\phi_{m}$ \\
\hline
\end{tabular}

also important to recall that in Part I some crucial changes were introduced into the WK model in order to allow for the exchange of sensible heat fluxes between the $\mathrm{ABL}$ and the deep troposphere through shallow cumulus entrainment and downdraft fluxes; entrainment $\alpha_{E}$ and downdraft parameters $\beta_{D}$ for these effects were introduced into the free troposphere moisture and first baroclinic temperature equations in Table 1 . These effects were absent in the original WK model (WK09) since sensible heat fluxes from the boundary layer are believed to be negligible for tropical convection over the ocean; however, for explaining diurnal cycle over land, these modifications are essential here.
The effects of the diurnal cycle of solar heating on the ABL are represented through the surface fluxes of moisture $\Delta_{s} q=q_{s}-q_{b}$ and temperature $\Delta_{s} \theta=\theta_{s}-\theta_{b}$, in the form of one-day periodic perturbations in the form of a half-sine function [see Part I and (2) below] added to the surface values $q_{s}$ and $\theta_{s}$, respectively:

$$
q_{s}(x, t)=\bar{q}_{s}+\hat{q}_{s}(x, t), \quad \theta_{s}(x, t)=\bar{\theta}_{s}+\hat{\theta}_{s}(x, t),
$$

where $t$ is time and $x$ is space.

To accommodate strong temperature inversions that on average characterize continental regions in the tropics (Fu et al. 1999; Fisch et al. 2004), we assume a cooler 
TABLE 2. Constants and parameters for the WK and KM models.

\begin{tabular}{|c|c|c|}
\hline Parameter & Value & Description \\
\hline$h_{b}, H_{T}, \delta$ & $500 \mathrm{~m}, 16 \mathrm{~km}, 0.031$ & ABL depth, free troposphere depth, ratio of $h_{b}$ to $H_{T}$ \\
\hline$Q_{R 1}$ & $1 \mathrm{~K}_{\mathrm{day}}{ }^{-1}$ & First baroclinic radiative cooling rate \\
\hline$Q_{R 2}$ & Determined at RCE & Second baroclinic radiative cooling rate \\
\hline$Q_{R b}$ & Determined by RCE & Boundary layer radiative cooling rate \\
\hline$\xi_{\tilde{S}}, \xi_{\mathcal{c}}$ & $8 / 3,0$ & Stratiform, congestus contribution to first baroclinic mode \\
\hline$\tilde{Q}, \tilde{Q}_{0}$ & $0.9,6.5$ & Background moisture stratification, contribution to barotropic vertical moisture advection \\
\hline$\tilde{\tilde{\lambda}}, \tilde{\alpha}$ & $0.8,0.1$ & Coefficient of $u_{2}$ in linear, nonlinear moisture convergence \\
\hline$m_{0}$ & Determined at RCE & Large-scale background downdraft velocity \\
\hline$\mu$ & 0.25 & Contribution of convective downdrafts to $M_{d}$ \\
\hline$\alpha_{s}, \alpha_{c}$ & $0.25,0.1$ & Stratiform, congestus adjustment coefficient \\
\hline$\alpha_{m}$ & 0.2 & Ratio of downdraft velocity $M_{d}$ to upward mass flux velocity $M_{u}$ at top of ABL \\
\hline$\tau_{R}, \tau_{D}$ & 75 days, 50 days & Rayleigh drag, Newtonian cooling time scale \\
\hline$\tau_{s}, \tau_{c}$ & $3 \mathrm{~h}, 1 \mathrm{~h}$ & Stratiform, congestus adjustment time scale \\
\hline$\tau_{\text {conv }}$ & $2 \mathrm{~h}$ & Convective time scale \\
\hline$\tau_{e}$ & Determined by RCE & Surface evaporation time scale \\
\hline$\tau_{T}$ & $8 \mathrm{~h}$ & Momentum entrainment time scale \\
\hline $\bar{Q}$ & Determined at RCE & Bulk convective heating at RCE \\
\hline$a_{1}, a_{2}$ & $0.45,0.55$ & Relative contribution of $\theta_{e b}, q$ to deep convection \\
\hline$a_{0}, a_{0}^{\prime}$ & $7,1.5$ & Dry convective buoyancy frequency in deep, congestus equilibrium \\
\hline$\gamma_{2}, \gamma_{2}^{\prime}$ & $0.1,2$ & Relative contribution of $\theta_{2}$ to deep, congestus heating \\
\hline$\alpha_{2}$ & 0.1 & Relative contribution of $\theta_{2}$ to $\theta_{e m}$ \\
\hline$\kappa$ & 2 & Ratio of $q_{t}$ to $q$ \\
\hline$C_{d}$ & 0.001 & Surface drag coefficient \\
\hline$u_{0}$ & $2 \mathrm{~m} \mathrm{~s}^{-1}$ & Strength of turbulent fluctuations \\
\hline$\theta^{-}, \theta^{+}$ & $2.5 \mathrm{~K}, 12.5 \mathrm{~K}$ & Moisture switch threshold values (for $7.5-\mathrm{K}$ inversion) \\
\hline
\end{tabular}

boundary layer at radiative-convective equilibrium (RCE) (consistent with nocturnal radiative cooling) while preserving the equilibrium temperature profile in the free troposphere (i.e., environmental lapse rate) identical to the case over the ocean where the temperature inversion is weak or nonexistent (see Fig. 1). Here we use $\Delta_{t} \bar{\theta}=$ $-7.5 \mathrm{~K}$ as our standard value for the temperature inversion over land, which is sufficient in magnitude to produce the desired afternoon peak in precipitation with a reasonable strength.

The equilibrium profiles of $\theta$ and $\theta_{e}$ used here for the land regime are plotted with dark lines in Fig. 1 (in the left and right panels, respectively) while the light lines represent the ocean reference profiles. It should be noted that in the original model the discrepancy between boundary layer and midtropospheric potential
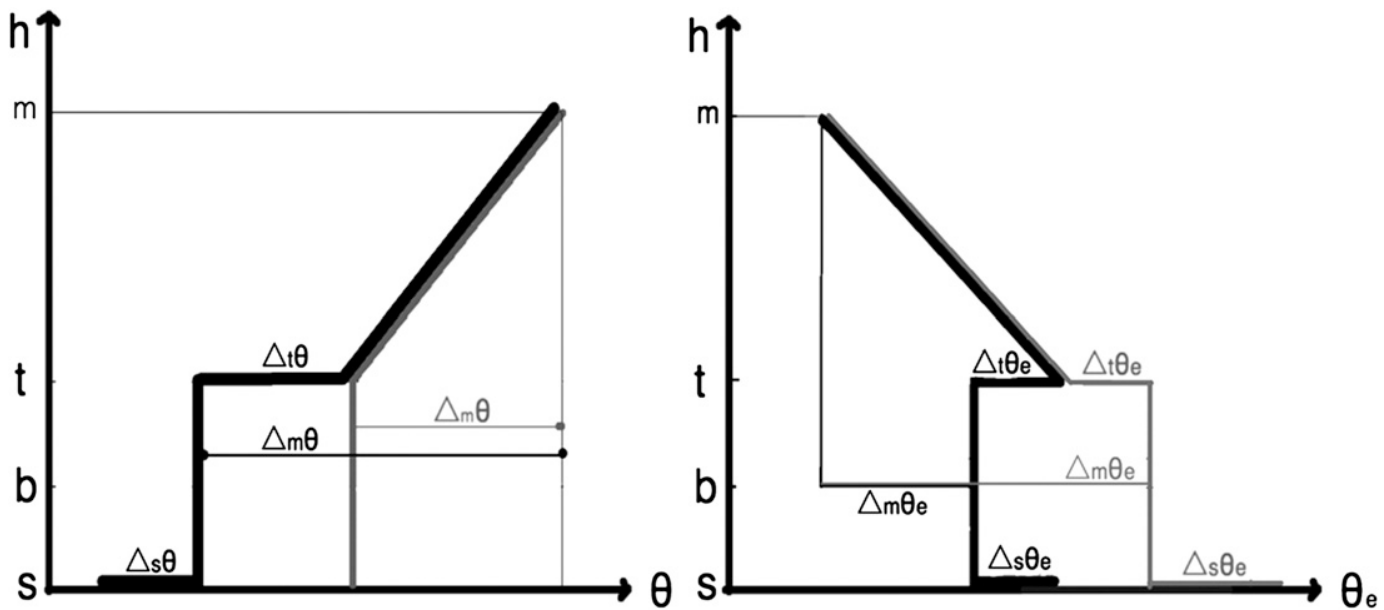

FIG. 1. (left) Potential and (right) equivalent potential temperature profiles for the WK model. The gray profiles denote the WK model in the ocean regime (as in WK08). Black profiles illustrate the effect of potential temperature inversion. Letters denote the surface (s), middle of the boundary layer (b), top of the boundary layer (t), and middle of the troposphere $(\mathrm{m})$. Note that the environmental lapse rate is preserved for the free troposphere. 
temperature $\Delta_{m} \bar{\theta}=\bar{\theta}_{b}-\bar{\theta}_{m}$ is constrained by the value of $\gamma \equiv-\Delta_{m} \bar{\theta} / \Delta_{m} \bar{\theta}_{e}$ given in Table 2. To preserve the original free troposphere lapse rate $X_{t}-X_{m}$ for both the moisture and potential temperature, the boundary layer potential temperature is cooled down to match the temperature inversion on top of the ABL. This results in $\Delta_{m} \theta_{e}=6.5 \mathrm{~K}$ for the standard case, which is in the range of observed values over the Amazon forest (Fu et al. 1999). Furthermore, $\Delta_{s} \bar{\theta}$ (which is set to zero over the ocean) is lowered by approximately the same amount as the inversion, which is consistent with the large Bowen ratios observed over land.

We recall that given the climatological values for the discrepancies $\Delta_{x} \bar{\phi}, x=s, m, t$ (for both $\phi=\theta$ and $\phi=q$, with $s$ indicating the surface, $m$ the middle troposphere and $t$ the top of the boundary layer) and the rate of radiative cooling $Q_{R, 1}^{0}$, the homogeneous RCE solution determines the values of the constants $Q_{R, 2}^{0}, Q_{R, b}, \bar{Q}, m_{0}$, and the surface evaporation time scale $\tau_{e}$ (WK09). As a consequence of the strong temperature inversion at the top of the ABL, the value of $\tau_{e}$ in the present land setup turns out to be around $2 \mathrm{~h}$. This may seem somewhat alarming because it is much smaller than the typical 8-h reference value used for the ocean case in Part I and in KM06, KM08, and WK09. However, according to similarity theory this decrease in the $\tau_{e}$ value can be associated with a larger roughness length scale over land terrain (Stull 1988). Additionally, the updraft mass flux velocity at the top of the ABL $M_{u}$, assumed to be proportional to the convective downdraft $D_{c}$ (Raymond 1995), is smaller, consistent with the inversion-dominated subsidence regime. This is accomplished by increasing the value of the parameter $\alpha_{m}$ from 0.2 to $0.7\left(\alpha_{m}=D_{c} / M_{u}\right)$. Overall, physically motivated choices of both $\alpha_{m}$ and $\Delta_{s} \bar{\theta}$ allow for a reasonable $\mathrm{ABL}$ cooling rate and evaporation time scale at RCE. While the choice of the parameters is not unique, the qualitative behavior of the system presented below is quite robust as discussed in section 4 .

As pointed out above, the value of $\Delta_{m} \bar{\theta}_{e}$ is lower because of the strong $\theta$ inversion as can be seen in Fig. 1. One critical variable that depends directly on the parameter $\Delta_{m} \bar{\theta}_{e}$ is the nonlinear switch $\Lambda$, which controls the transition between congestus and deep convection (KM06; KM08). To account for this drop in the standard value of $\Delta_{m} \bar{\theta}_{e}$, without disturbing the model physics, we lower the threshold values of $\Delta_{m} \theta_{e}$ that define the extremely dry and extremely moist limits by the amount of the inversion value, yielding, in the standard case, $\theta^{+}=$ $12.5 \mathrm{~K}$ and $\theta^{-}=2.5 \mathrm{~K}$, respectively.

In addition to the changes in inversion and boundary layer cumulus updrafts, the present version of the WK model has two other important new modifications. First, the congestus rain fraction is set to zero, since congestus clouds are rarely observed to precipitate over land (Casey et al. 2007). Second, the dry convective buoyancy parameter is doubled to $a_{0}=14$ to make deep convection more sensitive to variations in deep-troposphere potential temperature. The model's sensitivity to changes in those and other important parameters is discussed in section 4 .

\section{b. Diurnal cycle forcing, Bowen ratio, and Galilean transformation}

While diurnal variability over the ocean is driven by latent heat, over land it is due to both latent and sensible heat. Different heat capacities of land and ocean also influence the amount of energy available for the diurnal cycle at a given time of the day but we neglect this effect here for the sake of simplicity. A useful quantity in the study of the diurnal cycle forcing over land is the ratio of fluxes of sensible heat and latent heat (produced by solar heating) known as the Bowen ratio and denoted here by $\beta$ (not to be confused with the gradient of the Coriolis force, which is ignored here). Over the ocean, the Bowen ratio is typically less than 0.1 (Hsu 1998; Sadhuram et al. 2001) because latent heat is the primary response of the ocean surface to solar heating. This is exploited in the original derivation of the multicloud model by Khouider and Majda (KM06; KM08) where a single equation for the $\mathrm{ABL} \theta_{e}$ was used to represent the boundary layer dynamics. Over desert regions, the Bowen ratio can reach values of up to $\beta=$ 10 (Chapin et al. 2002). The scarcity of moisture and high surface albedo makes sensible heat the dominant response of the desert terrain to solar heating. In this paper, we are mostly concerned with the case of equatorial forests where the large transpiration and soil moisture allows for Bowen ratios in the range of $\beta=0.2-0.4$ (Chapin et al. 2002).

In the present model, as shown in (1), the effect of solar radiation is represented through perturbations of both the surface temperature and surface moisture variables. Here the amplitude of the diurnal variation in surface temperature is inferred directly from observations (Yang and Slingo 2001) while a Bowen ratio that is appropriate for the Amazon forest (Chapin et al. 2002) is used to approximate the corresponding moisture surface flux perturbation. Accordingly, the perturbations of surface moisture and temperature used in the present study have a maximum amplitude of 10 and $4 \mathrm{~K}$, respectively. It is worth noting that $4-\mathrm{K}$ maximum amplitude of surface temperature perturbation is comparable to the typical values used in global climate simulations for the Amazon region (Lin et al. 2000). While the Bowen ratio is appropriate for the equatorial forest, the amplitude of the variation is deliberately conservative. In fact, it makes variations in the boundary layer equivalent potential temperature comparable to the perturbation used in the 
ocean case addressed in Part I to facilitate comparison. It should be further noted that the perturbation used in the ocean case was calculated from near-maximum values of observed SST diurnal variation while the solutions with smaller perturbations showed the same qualitative behavior. In the present case, we chose the amplitude of the perturbation conservatively to reduce the set of parameter changes when going back and forth between the ocean and land scenarios. In the continental regime, the results presented in this paper are qualitatively similar to the cases with higher perturbation amplitudes. In fact, higher total perturbations produce higher afternoon precipitation maxima with similar cycles (see section 4).

As in Part I, the same half-sine perturbation profile is used here for both surface latent and sensible heat diurnal variations. It can be justified by considering observed sensible and latent heat flux where the small effects of soil thermal inertia are ignored.

$$
\hat{\theta}_{e b}^{*}(x, t)=\hat{\theta}_{e b, \max }^{*}\left\{\begin{array}{lll}
\frac{\pi}{\pi-1}\left[\sin (x+c t)-\frac{1}{\pi}\right] & \text { if } & x+c t \bmod (2 \pi)<\pi \\
-\frac{1}{\pi-1} & \text { if } & x+c t \bmod (2 \pi)>\pi
\end{array} .\right.
$$

Here we recall the Galilean $\theta^{*}$ transformation applied to the governing equations that facilitates the analysis. The peak of solar energy traverses the $40000-\mathrm{km}$ perimeter of the earth every $24 \mathrm{~h}$, with constant speed of $c=$ $463 \mathrm{~m} \mathrm{~s}^{-1}$. This naturally leads to a reference frame aligned with this constant velocity motion. A Galilean transformation $x_{g}=x+c t, \tau=t$ is thus applied to the governing equations where the effects of waves and other nonhomogeneities are ignored (Part I). Notice that variations in $x_{g}$ can be interpreted accordingly as relating to different locations on the equator during the same time of the day or relating to a fixed location at different times of the day [i.e., local solar time (LST)]. The latter is adopted throughout the paper just as in Part I. To focus on the diurnal variation of the background climatology, without consideration of wave activity and other flow complexities, we seek solutions $U=U\left(x_{g}\right)$ that do not depend on the new time variable $\tau$. This results in an ODE system with periodic forcing as reported in Part I. This system is reproduced here for the sake of completeness.

$$
\begin{aligned}
c \bar{u}^{\prime}= & -\bar{u} \bar{u}^{\prime}-p_{0}^{\prime}-\bar{u}^{\prime}\left(\bar{u}-u_{b}\right)+\frac{E_{u}}{H_{T}} \Delta_{t} u \\
c u_{j}^{\prime}= & -\bar{u} u_{j}^{\prime}-u_{j} \bar{u}^{\prime}-\theta_{j}^{\prime}-\frac{u_{j}}{\tau_{R}}+\frac{\sqrt{2}}{T_{T}} \delta_{b} \Delta_{t} u \\
c \theta_{1}^{\prime}= & -\bar{u} \theta_{1}^{\prime}+u_{1}^{\prime}-\sqrt{2} \bar{u}^{\prime}+\frac{\pi}{2 \sqrt{2}}\left[P+\alpha_{E} \frac{E}{H_{T}} \Delta_{t} \theta+\beta_{D}\left(\frac{M_{d}}{H_{T}}+\bar{u}^{\prime}\right) \Delta_{m} \theta\right]-Q_{R 1}-\frac{\theta_{1}}{\tau_{D}} \\
c \theta_{2}^{\prime}= & -\bar{u} \theta_{2}^{\prime}+\frac{1}{4} u_{2}^{\prime}-\frac{\sqrt{2}}{4} \bar{u}^{\prime}-H_{s}+H_{c}-Q_{R 2}-\frac{\theta_{2}}{\tau_{D}} \\
c q^{\prime}= & -\bar{u} q-\left[\left(u_{1}+\delta u_{2}\right) q+\left(u_{1}+\tilde{\lambda} u_{2}\right) \tilde{Q}-\bar{u} \tilde{Q}_{0}\right]^{\prime}-P+\frac{E}{H_{T}} \Delta_{t}\left[\left(1-\alpha_{E}\right) \theta+q_{b}\right] \\
& +\left(\frac{M_{d}}{H_{T}}+\bar{u}^{\prime}\right) \Delta_{m}\left[\left(1-\beta_{D}\right) \theta+q_{b}\right] \\
c q_{b}^{\prime}= & -u_{b} q_{b}^{\prime}-\frac{E}{h_{b}} \Delta_{t} q-\frac{M_{d}}{h_{b}} \Delta_{m} q+\frac{1}{\tau_{e}} \Delta_{s} q \\
c \theta_{b}^{\prime}= & -u_{b} \theta_{b}^{\prime}-\frac{E}{h_{b}} \Delta_{t} \theta-\frac{M_{d}}{h_{b}} \Delta_{m} \theta+\frac{1}{\tau_{e}} \Delta_{s} \theta-Q_{R b} \\
c u_{b}^{\prime}= & -u_{b} u_{b}^{\prime}-p_{b}^{\prime}-\frac{E_{u}}{h_{b}} \Delta_{t} u-\frac{C_{d} U}{h_{b}} u_{b} \\
p_{0}= & p_{b}+\sqrt{2}\left(\theta_{1}+\theta_{2}\right)+\frac{\pi}{2} \delta_{b} \theta_{b} \\
\left(1+\delta_{b}\right) p_{b}^{\prime}= & 2 \delta_{b} \bar{u}^{\prime}\left(\bar{u}-u_{b}\right)-\sqrt{2}\left(\theta_{1}^{\prime}+\theta_{2}^{\prime}\right)-\frac{\pi}{2} \delta_{b} \theta_{b}^{\prime}-\delta_{b} \frac{C_{d} u_{0}}{h_{b}} u_{b}
\end{aligned}
$$


Here the prime denotes the derivative with respect to the variable $x_{g}$, which is the only independent variable in this system, while the dependent variables are as in Table 1 . To be more explicit, note that the periodic forcing in (3) is represented in the jumps $\Delta_{s} \theta_{b}$ and $\Delta_{s} q_{b}$ that depend directly on the diurnal perturbations of the surface temperature $\hat{\theta}_{s}\left(x_{g}\right)$ and the saturation moisture $\hat{q}_{s}\left(x_{g}\right)$. These perturbations are plotted in the bottomright corner of Fig. 2.

As mentioned in Part I, it is not possible to write (3) as an ODE system in explicit form. A second-order accurate one-step finite difference method (described in the appendix of Part I) is used to solve these equations. The stability of the resulting periodic solutions is checked via direct numerical integration with initial conditions characterized by small random perturbations about the periodic solution.

\section{Model results and comparison to observations: The land case}

\section{a. The typical stable 1-day periodic solution}

As is the case of the ocean in Part I, our search for stable periodic solutions is guided by linear stability analysis of the homogeneous RCE steady state. The results of linear stability analysis are similar to what is presented in Part I and are therefore omitted here. As already pointed out in Part I, while the direct application of Floquet theory to the WK equations is not feasible, nonlinear stability of periodic solutions can be inferred by direct numerical evolution of the system with a small random perturbation added at initial time. All solutions presented in this paper are nonlinearly stable, even though in some extreme parameter regimes unstable solutions do exist, with a structure similar to the unstable periodic and chaotic solutions described in Part I. Notice, however, that the smallness of the velocity components, because of their weak coupling to the convective heating in this climatological setting where wave activity is ignored, as pointed out in Part I, can be exploited here to reduce the system in (3) by asymptotic expansion into a system that is amenable to an explicit formulation $\left[y^{\prime}=\right.$ $f(x, y)]$ for which Floquet theory is applicable. This is done in section $3 b$.

A typical stable periodic solution associated with the diurnal cycle forcing over land is plotted in Fig. 2, for the case of a relatively moist RCE background state with $\bar{\theta}_{e b}-\bar{\theta}_{e m}=6.5 \mathrm{~K}$ and a $7.5-\mathrm{K}$ temperature inversion capping the boundary layer. The rest of the model parameters assume the standard values reported in Table 2. The response of the boundary layer potential temperature and moisture are shown in the bottomright panel of Fig. 2. Notice that while $\theta_{b}$ is more or less in phase with the imposed diurnal heating, which peaks at 1200 LST, the boundary layer moisture perturbation $q_{b}$ has a significant lag of about $2 \mathrm{~h}$, consistent with the $\theta_{e b}$ fluctuations seen in Part I for the ocean case. As expected, the velocity components of the periodic solution, shown in the top-left panel of Fig. 2, are extremely weak.

The most striking feature of the solution is a prominent afternoon peak displayed by the precipitation plot on the bottom-left panel of Fig. 2, consistent with the deep convective heating $H_{d}$ curve in the right panel of the third row. It is important to recall here that we set $f_{c}=0$ for the land case and therefore congestus heating does not contribute to the precipitation. In fact, the lack of precipitation from congestus clouds permits a rapid buildup of moisture (see Fig. 2, left panel of the third row) in early afternoon, which precedes the deep convective maximum. The afternoon deep convective precipitation maximum is consistent with the surface observations of the diurnal cycle of precipitation over the Amazon forest (Machado et al. 2004) where the peak of precipitation is observed between 1400 and 2000 LST depending on the geographical location and season. The peak is also consistent with satellite data-based studies found in Lin et al. (2000), where peak of precipitation is observed around 1500 LST (the minimum occurs in midmorning). It should be noted that the structure of diurnal precipitation over the tropical continents is highly dependent on the geographical location, terrain, and vegetation type as well as the season. The simplicity of our model may not warrant a detailed comparison with observations, although its qualitative behavior is consistent with land precipitation patterns in the tropics. In particular, the morning local maximum of the precipitation (see Fig. 4) is not observed in nature. This discrepancy with observations may be regarded as a serious shortcoming of the present model, but this is presumably due to some important physical processes that are not (well) represented in the present model. Such mechanisms include thermal inertia of the land surface and cloud radiative forcing that are both ignored in the model. Nonetheless, the relatively much stronger afternoon precipitation maximum and the midmorning precipitation minimum, two robust features of the model that are qualitatively similar to the observations, constitute a very interesting and encouraging result. The effects of thermal inertia, cloud forcing, and other relevant processes will be incorporated into the model and reported elsewhere in the near future.

We conclude this section by noting that the imposed strong inversion layer for the time-averaged background corresponding to $\Delta_{t} \theta=-7.5 \mathrm{~K}$ and $\Delta_{t} q=5 \mathrm{~K}$, resulting in an effective inversion of $\Delta_{t} \theta_{e}=-2.5 \mathrm{~K}$, is somewhat compensated by the diurnal solution shown on the 

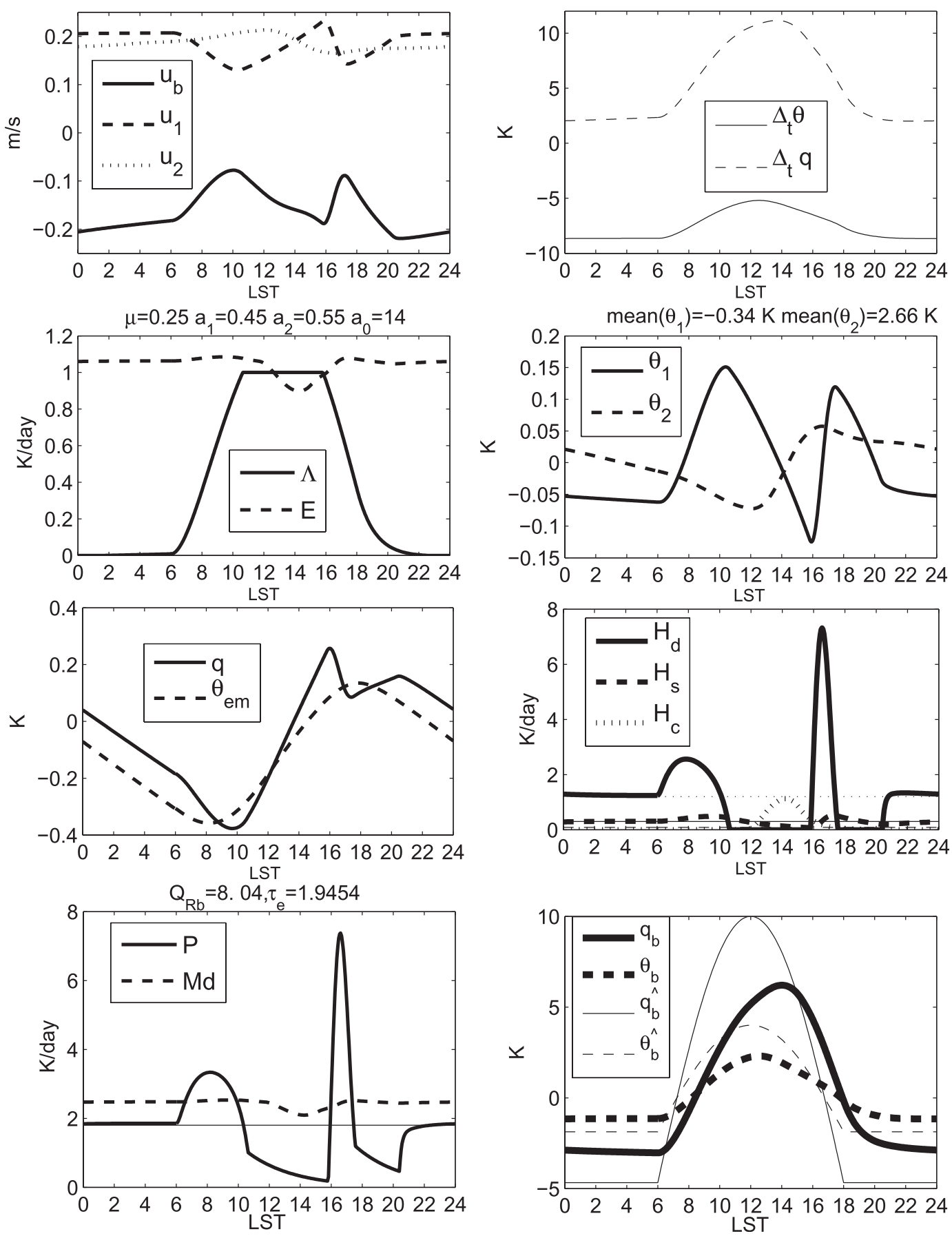

FIG. 2. Stable periodic response of the KM model to a diurnal cycle forcing for the case of a moist background corresponding to $\bar{\theta}_{e b}-\bar{\theta}_{e m}=6.5 \mathrm{~K}$ (comparable to $\bar{\theta}_{e b}-\bar{\theta}_{e m}=14 \mathrm{~K}$ of the original WK model). The rest of the parameters are fixed to their standard values in Table 2.

top-right panel of Fig. 2, which displays an overall $\Delta_{t} \theta$ that varies roughly between -8 and $-5 \mathrm{~K}$ and a perturbation $\Delta_{t} q$ that varies between 2 and $10 \mathrm{~K}$, with the latter values corresponding to the afternoon precipitation maximum. This corresponds to an effective inversion varying between roughly $-6 \mathrm{~K}$ at night to a slightly positive value (i.e., a conditionally unstable profile) during the afternoon. While this is consistent with the model physics, there is no observational evidence for such strong $\Delta_{t} \theta$ jumps during deep convective events over land or over the ocean. This may be an artifact of the model's fixed boundary layer height, among other possibilities. 


\section{b. Single-column model approximation}

As pointed out above, the smallness of the velocity components in Fig. 2 suggests that spatial dependence may not be critical for the diurnal cycle response of the background climate when wave oscillations are ignored. To confirm this here we neglect the spatial dependence by zeroing the velocity vectors wherever they appear in (3). This leads to a simple column model system that can be written in explicit form as follows:

$$
\begin{aligned}
\theta_{1}^{\prime}= & \frac{\pi}{2 \sqrt{2}}\left(P+\alpha_{E} \frac{E^{c}}{H_{T}} \Delta_{t} \theta+\beta_{D} \frac{M_{d}^{c}}{H_{T}} \Delta_{m} \theta\right)-Q_{R 1}-\frac{\theta_{1}}{\tau_{D}} \\
\theta_{2}^{\prime}= & -H_{s}+H_{c}-Q_{R 2}-\frac{\theta_{2}}{\tau_{D}} \\
q^{\prime}= & -P+\frac{E^{c}}{H_{T}} \Delta_{t}\left[\left(1-\alpha_{E}\right) \theta+q_{b}\right] \\
& +\frac{M_{d}^{c}}{H_{t}} \Delta_{m}\left[\left(1-\beta_{D}\right) \theta+q_{b}\right] \\
q_{b}^{\prime}= & -\frac{E^{c}}{h_{b}} \Delta_{t} q-\frac{M_{d}^{c}}{h_{b}} \Delta_{m} q+\frac{1}{\tau_{e}} \Delta_{s} q \\
\theta_{b}^{\prime}= & -\frac{E^{c}}{h_{b}} \Delta_{t} \theta-\frac{M_{d}^{c}}{h_{b}} \Delta_{m} \theta+\frac{1}{\tau_{e}} \Delta_{s} \theta-Q_{R b}
\end{aligned}
$$

Here, for the column model equations, the downdraft and entrainment mass fluxes are respectively reduced to $M_{d}^{c}=D_{c}^{+}$and $E^{c}=\left(M_{u}-M_{d}\right)^{+}$.

The resulting boundary value problem is solved by the standard BVP method of Matlab used in Part I. Figure 3 presents the solution of the column model for the same parameter regime as in Fig. 2. The resulting precipitation profiles of the full and column model are nearly identical. Under close scrutiny, however, we can discern small differences in state variables. For example, the free tropospheric moisture of the full model solution is slightly higher during the period of high congestus activity. Although negligible, this small difference is clearly associated with congestus preconditioning via the induced large-scale low-level convergence. However, the resulting difference in maximum precipitation is less than $0.15 \mathrm{~K}$ $\mathrm{day}^{-1}$. Thus, for this climate background case, where wave activity is ignored, it is reasonable to state that the column model formulation is sufficient to reproduce the main features of the model's response to diurnal cycle forcing and that large-scale moisture convergence is of secondary significance during the moistening phase.

Moreover, as already pointed out, another important advantage of the column model (4) is that it is suitable for Floquet stability analysis, as done for the Khouider and Majda (KM) model in Part I. When the Floquet analysis is applied to the solution in Fig. 3, it is found that the latter is asymptotically stable with the associated largest Floquet multiplier being approximately 0.85 . This is consistent with the numerical predictions of the previous section according to which the solution is nonlinearly stable.

While the column model is extremely simple, the incremental numerical cost of solving the full model equations in (3) is not prohibitive. Thus, for the sake of completeness, in the remaining of the paper the full model equations are used instead of the simpler column model version in (4). However, the latter is used to confirm that all the corresponding solutions are Floquet asymptotically stable, although the results are not reported here.

\section{c. The physical mechanism of diurnal cycle of precipitation over land}

The underlying physical mechanism and dynamical behavior associated with the solutions in Fig. 2 is explained here in terms of the interactions of the three cloud types with the periodically forced boundary layer dynamics. It is evident from the plots in Fig. 2 that the sudden acceleration of the morning deep convection is driven by the rise of $\theta_{e}$ in the ABL (i.e., CAPE at sunrise). However, this morning deep convection is followed by a rapid warming and drying of the middle troposphere, which disfavors deep convection. The warming is due to both deep-convective heating and mixing of ABL sensible heat due to detrainment of shallow cumulus clouds, through the effects of the sensible heat entrainment parameter (set to $\alpha_{E}=1 / 3$ here).

The dry and warm environment of the early afternoon combined with abundance of the low-level CAPE $Q_{c}$ favors congestus heating that slightly warms the lower troposphere and cools the upper troposphere, thus delaying the reinitiation of deep convection. Meanwhile, the midtroposphere moisture builds up because of shallow cumulus entrainment and detrainment fluxes. The afternoon congestus also can moisten the middle atmosphere through low-level moisture convergence (KM06; KM08) but it is unlikely that this is the main mechanism at work here since the velocity components are very weak. Shortly after 1500 LST, the atmosphere reaches relative humidity that allows for deep convection. The massive amount of accumulation of CAPE is then discharged quickly in the moist-preconditioned troposphere and results in the explosive afternoon deep convection episode, just before sunset.

After sunset, the tropospheric moisture recovers quickly. However, because of the relative increase in precipitation, the ABL moisture starts dropping below its equilibrium value shortly after 1800 LST because of downdrafts. At this time, the troposphere nearly reaches a RCE state characterized by a moderate precipitation rate of approximately $1.5 \mathrm{~K} \mathrm{day}^{-1}$ (roughly balancing the imposed radiative cooling), which is a plateau value between roughly 

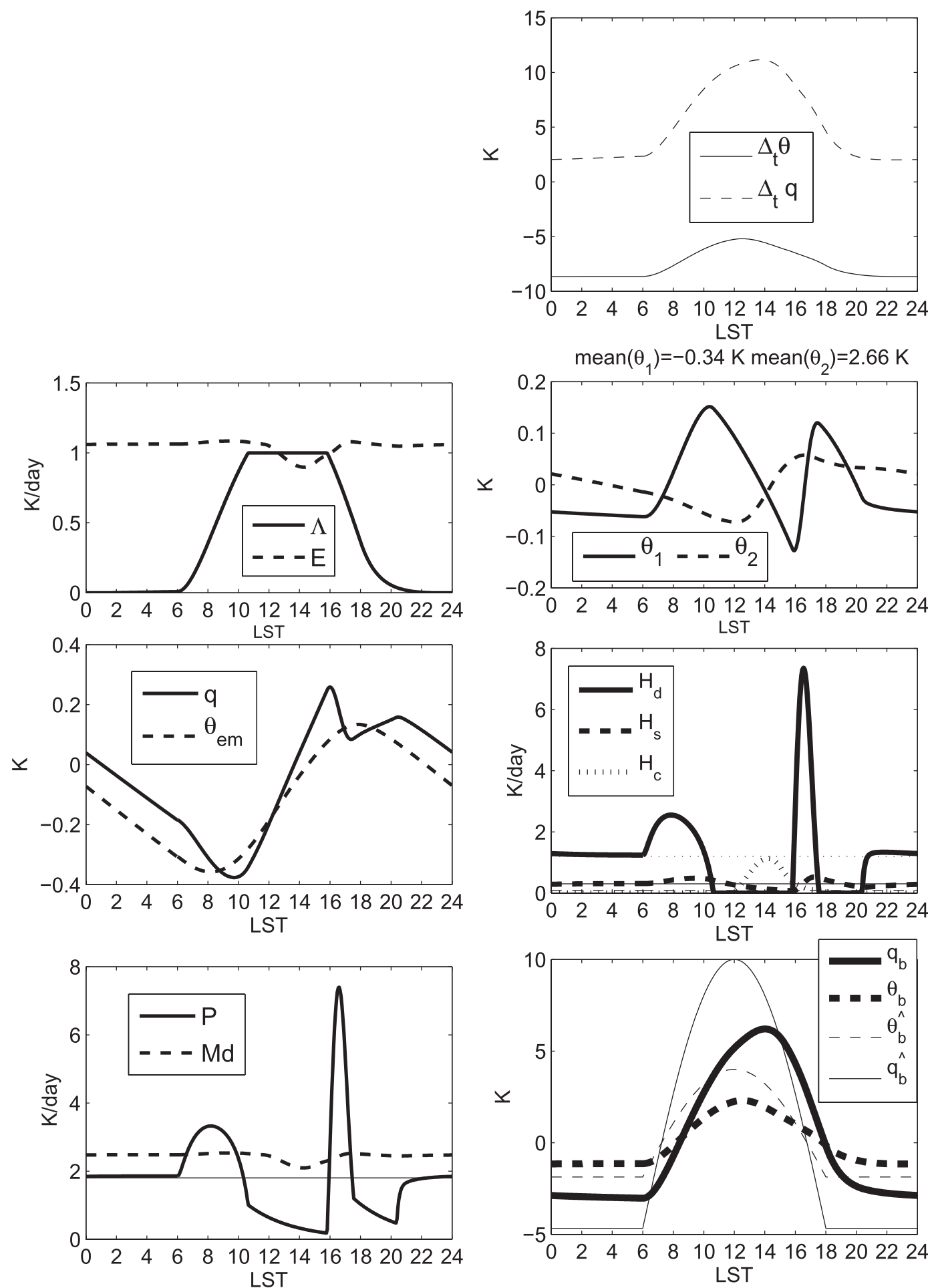

FIG. 3. Floquet stable periodic response of the column model version of WK model in (4) to a diurnal cycle forcing. The parameter regime is identical to Fig. 2.

2000 and 0600 LST. Note a significant decrease of potential temperature $\theta_{1}$ during the evening between roughly 1800 and 2000 LST due to the imposed radiative cooling of $1 \mathrm{~K} \mathrm{day}^{-1}$. The sudden rise in CAPE in the morning combined with the relatively cold troposphere allows for deep convection to rise beyond its overnight equilibrium level at sunrise and closes the cycle described above. 
The diurnal cycle of precipitation over land can be divided into a cycle of five phases somewhat similar to the ocean case reported in Part I although with very different physics and different timing. 1) A persistent phase of overnight RCE level precipitation where convective heating balances the imposed longwave radiation, which prevails roughly between 2000 and 0600 LST, is followed by 2) a CAPE buildup and a tropospheric warming phase associated with the early morning warming of the boundary layer, which expands between 0600 and roughly 1000 LST and is characterized by a significant rise in precipitation that accelerates the drying and warming of the middle troposphere. Then, 3) a rapid and intense (re)moistening phase of the relatively warm troposphere to hold significant amounts of vapor starts at around 1000 LST. This is accompanied by congestus heating that favors further moisture storage. 4) An explosive precipitation peak phase starts at 1600 LST. It quickly dries the troposphere and consumes CAPE by warming the troposphere and dies slightly before 1800 LST. This phase is followed by 5) a second (re)moistening episode that establishes the moisture level to sustain the overnight precipitation from phase 1 .

As demonstrated in the next section though a series of sensitivity tests, the main physical mechanisms that control this five-phase cycle of precipitation over land are due to the combination of four main processes that characterize the present model setup, namely 1) a strong temperature inversion on the top of the ABL of about $7.5 \mathrm{~K}, 2$ ) shallow cumulus entrainment and downdraft fluxes of sensible heat corresponding to $\alpha_{E}=\beta_{D}=1 / 3$, 3 ) a strong Bowen ratio of about $\beta=0.4$, and 4) nonprecipitating congestus heating that allows a rapid and intense moistening during midday and early afternoon $\left(\xi_{c}=0\right.$ for the standard parameters in Table 2).

\section{Sensitivity to parameters and comparison to the ocean case}

\section{a. Moist and dry RCE states, inversion, and entrainment}

The prototypical solution associated with the standard parameters in Table 2 corresponds to a mildly moist RCE atmosphere $\bar{\theta}_{e b}-\bar{\theta}_{e m}=6.5 \mathrm{~K}$ that, in terms of the switch function, corresponds to $\bar{\theta}_{e b}-\bar{\theta}_{e m}=14 \mathrm{~K}$ in the ocean setup in Part I. Recall that for the ocean case in Part I, dry tropospheric RCEs are characterized by a strong secondary afternoon congestus precipitation peak for $\bar{\theta}_{e b}-\bar{\theta}_{e m} \geq 16 \mathrm{~K}$. However, this is not the mechanism at work in the development of a strong afternoon precipitation peak here, since congestus clouds do not precipitate in the land setup. However, the magnitude of the
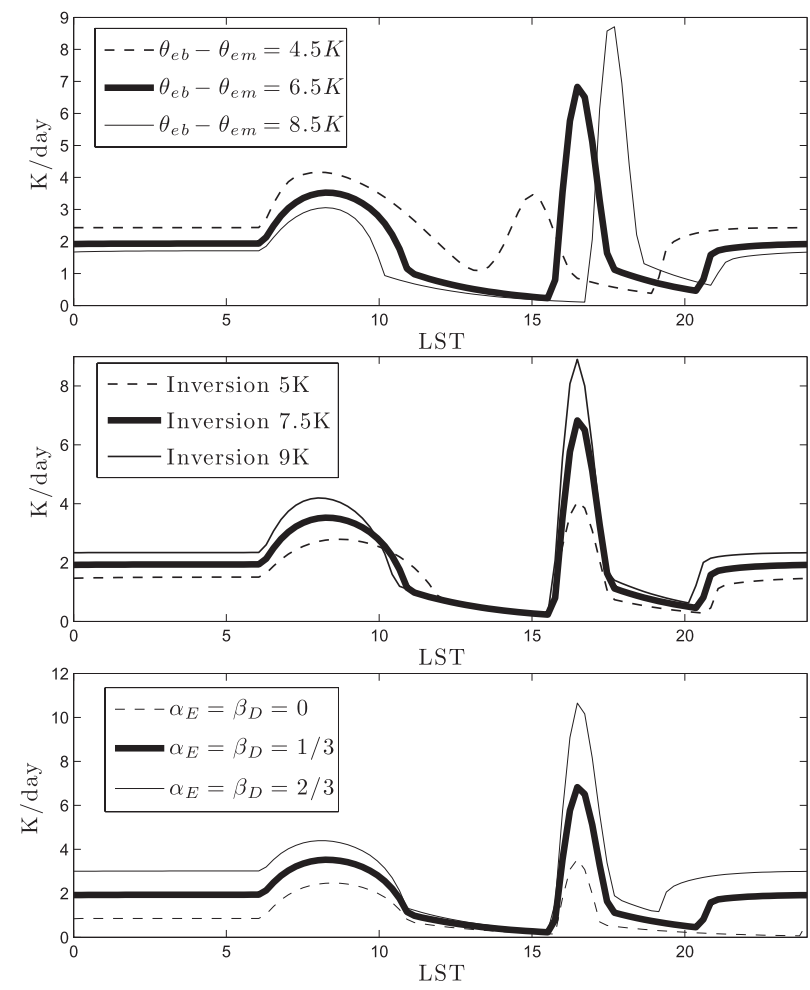

FIG. 4. Sensitivity of precipitation to variation in (top) $\Delta \theta_{e}$, (middle) inversion, and (bottom) $\alpha_{E}$. The standard case is shown as a thick line while the rest of the parameters are fixed to their standard values in Table 2.

afternoon precipitation does increase with a drier RCE state, consistent with the ocean case.

The effect of the variation of the background moisture on the diurnal cycle of precipitation is depicted in the top panel of Fig. 4, which compares the standard case to a moister and a drier RCE corresponding to $\bar{\theta}_{e b}-\bar{\theta}_{e m}=4.5$ and $8.5 \mathrm{~K}$, respectively. Similar to the ocean case, the moist RCE case allows for strong deep convection in the morning, which uses up most of the moisture. As a consequence, the secondary afternoon precipitation peak is weaker in magnitude despite the larger daytime CAPE magnitude. On the other hand, a drier RCE atmosphere is not favorable to strong morning convection due to low relative humidity. Instead, congestus clouds (favored by the nonlinear moisture switch) use up low-level CAPE and allow for upper troposphere cooling while the abundant ABL latent heat flux moistens the troposphere in the early afternoon through entrainment mixing associated with shallow cumulus clouds. Since the troposphere is drier it takes longer to moisten, resulting in a delay in the afternoon precipitation peak. In fact, the dry case allows for both higher afternoon and lower morning precipitation, not reported in detail here where we omit extreme values. 
Nonetheless, the evidence of this behavior can be found in observations (Machado et al. 2004). The moist RCE of $\bar{\theta}_{e b}-\bar{\theta}_{e m}=6.5 \mathrm{~K}$ is typical for the atmosphere above primal forest of Belem, for example, during the rainy season, while the drier case $\bar{\theta}_{e b}-\bar{\theta}_{e m}=8.5 \mathrm{~K}$ corresponds to the dry season. An evening shift in precipitation maximum during the dry season can be observed in Fig. 8 of Machado et al. (2004).

At this point one can be misled to believe that the afternoon deep convection results purely from a drier atmospheric profile setting at RCE and a lack of congestus precipitation invoked in the model by setting $f_{c}=0$. However, these two mechanisms by themselves result only in a small afternoon deep convective peak. Other changes in parameters - namely a strong temperature inversion at the top of the $\mathrm{ABL}$, a large Bowen ratio, and nonzero entrainment and downdrafts fluxes of sensible heat $\left(\alpha_{E} \neq 0, \beta_{D} \neq 0\right)$-are all necessary to produce the observed strong afternoon precipitation, as demonstrated below.

The sensitivity of the precipitation cycle to the strength of the temperature inversion is presented in the middle panel of Fig. 4. Strong inversions are frequently encountered above the ABL of the Amazon forest (Fu et al. 1999). The inversion is a maximum in the morning and acts as a barrier inhibiting deep convection. In observations (Fu et al. 1999), it gets eroded during the day because of surface latent and sensible heat fluxes. Consistently, this is roughly what is seen in the top-right panel of Fig. 2 for the multicloud model.

We recall that the WK model is altered to include the redistribution of the entrainment and downdraft fluxes between the free tropospheric moisture and the potential temperature to allow penetration of sensible heat fluctuation through the top of the boundary layer interface. For the ocean case, discussed in Part I, these effects are negligible because of the weak Bowen ratio and weak temperature inversion. The inclusion of strong inversion here allows the boundary layer potential temperature fluctuation to impact significantly the temperature of the free troposphere through the first baroclinic temperature mode. Thus, ABL sensible heat warms the deep atmosphere during the day and inhibits deep convection.

Sensitivity tests of the diurnal cycle of precipitation due to changes in the entrainment $\alpha_{E}$ and downdraft parameters $\beta_{D}$ are depicted in the bottom panel of Fig. 4. No exchange of sensible heat at the top of the $\operatorname{ABL}\left(\alpha_{E}=\alpha_{D}=0\right)$ results in a colder midtroposphere (i.e., higher relative humidity with a small amount of moisture) and thus a stronger and more efficient morning deep convection. This scenario still creates an afternoon peak but it is comparable in amplitude to the morning convection due to the smaller moisture content.
High values of the entrainment and downdraft parameter $\left(\alpha_{E}=\beta_{D}=2 / 3\right)$ allow faster warming of the atmosphere by ABL sensible heat perturbation (which precedes the moisture fluctuation) and permits higher moisture loading and results in a much stronger afternoon precipitation peak.

Note that changes in RCE dryness, inversion strength, and entrainment and downdraft parameters are accompanied by an adjustment of the ABL cumulus updraft parameter $\alpha_{m}$ and $\Delta_{s} \bar{\theta}$ in order to preserve a comparable $Q_{R b}$ and $\tau_{e}$. Note that while the magnitude of the RCE precipitation changes, the above analysis is based on the relative sizes of morning and afternoon precipitation peaks. A moister troposphere results in a higher overnight RCE precipitation rates.

\section{b. Bowen ratio and dry buoyancy frequency}

A Bowen ratio of $\beta=0.4$ is used here as a standard value to represent a typical case of precipitation over tropical forests (Fu et al. 1999). The sensitivity tests due to changes in the Bowen ration are depicted in the top panel of Fig. 5. Note that the case $\beta=0$ is similar to the ocean scenario reported in Part I, characterized by a dominant morning precipitation peak, despite the fact that both the strong inversion and sensible heat entrainment and downdrafts are activated. The ABL potential temperature variation is very small and therefore convection is the sole source of heating of the deep troposphere. The morning deep convection dries the troposphere without a significant warming from sensible heat that would allow a rapid storage of moisture. Thus, the subsequent afternoon deep convection peak is weaker. Meanwhile, the higher Bowen ratio $(\beta=0.8)$ allows for faster morning warming of the troposphere. Therefore, morning deep convection stops earlier because of additional heating by sensible heat entrainment, which at the same time allows for significant storage of moisture around noon and early afternoon. Therefore, the afternoon peak is larger in magnitude and is slightly delayed since it takes longer for the warmer troposphere to cool down by the fixed radiation. The higher precipitation peaks, occurring at higher Bowen ratios, are due to larger total perturbations of surface fluxes, which result in higher CAPE anomalies.

The bottom panel of Fig. 5 displays sensitivity tests to variations in the dry buoyancy parameter $a_{0}$, used in the closure of the deep convection potential $Q_{d}$ (see Table 1 ), so that a colder middle troposphere facilitates deep convection by allowing saturation at weaker mixing ratios (Khouider and Majda 2006a). Because of the emphasis on the penetrative sensible heat flux in this paper, we choose a higher value of convective buoyancy parameter $\left(a_{0}=14\right)$ as the standard case, whereas it is set to $a_{0}=7$ for the ocean study in Part I. 

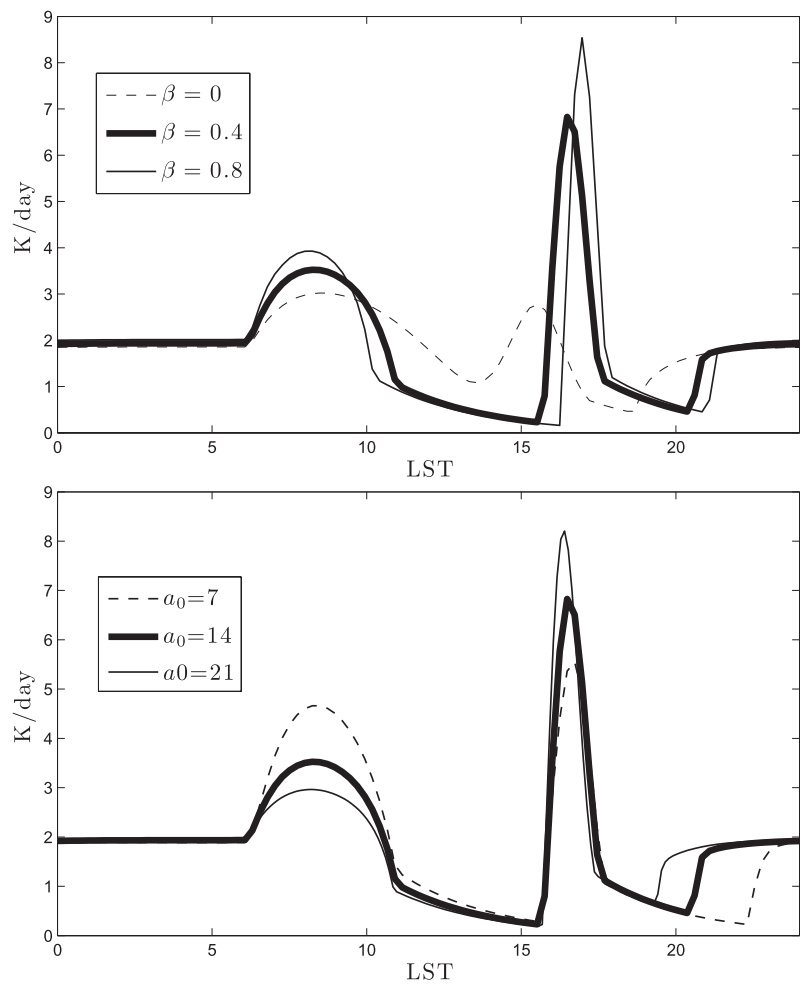

FIG. 5. Sensitivity of precipitation to variation in (top) $\beta$ and (bottom) $a_{0}$. The standard case is shown as a thick line while the rest of the parameters are fixed to their standard values in Table 2.

From the bottom panel of Fig. 5, we see that, as expected, higher $a_{0}$ values result in higher afternoon and lower morning precipitation peaks and vice versa for lower $a_{0}$. The higher $a_{0}$ values make deep convection more sensitive to temperature variations; thus, the rise in the ABL potential temperature perturbation successfully reduces the amplitude of the morning deep convective precipitation and allows storage of greater amounts of moisture to be available for the afternoon precipitation, as already anticipated.

\section{c. Sensitivity to stratiform and congestus rain fractions}

Recall that in KM08 the rain fractions due stratiform and congestus cloud types are given as averages relative to variations in the moisture switch function by $f_{s}=$ $\xi_{s} \alpha_{s}\left(1+\xi_{s} \alpha_{s}+\xi_{c} \alpha_{c}\right)^{-1}$ and $f_{c}=\xi_{c} \alpha_{c}\left(1+\xi_{s} \alpha_{s}+\xi_{c} \alpha_{c}\right)^{-1}$. The standard values used in this paper, $f_{s}=0.4$ and $f_{c}=0$, are chosen in accordance with the observational data according to which congestus clouds contribute very little to the total precipitation over land, while stratiform and deep convective precipitation contribute about $40 \%$ and $60 \%$, respectively (Schumacher and Houze 2003; Takayabu et al.2010). Stratiform rain fraction is observed to vary significantly with the geographic location all over
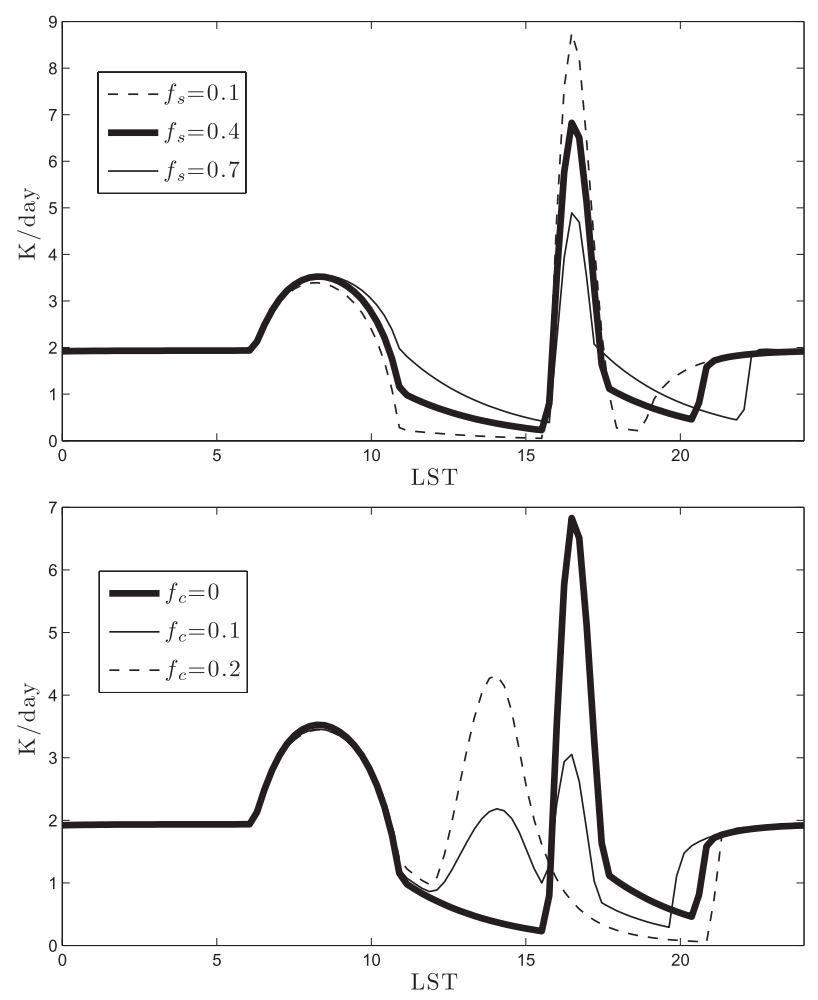

FIG. 6. Sensitivity of precipitation to variation in (top) $f_{s}$ and (bottom) $f_{c}$. The standard case is shown as a thick line while the rest of the parameters are fixed to their standard values in Table 2 .

the tropics. However, land regions tend to have lower stratiform rain fractions and higher deep convective activity. This is particularly the case over central Africa where stratiform rain accounts for about $20 \%-30 \%$ of the total precipitation. However, the central Amazon region has an average stratiform precipitation of about $30 \%-$ $40 \%$ (Casey et al. 2007), which motivated our standard value $f_{s}=0.4$

Sensitivity of the model to the stratiform rain fraction $f_{s}$ is presented at the top panel of Fig. 6. We note that with lower stratiform rain fraction the afternoon peak in total precipitation is larger. Recall that in the multicloud model, the stratiform clouds lag deep convection, which suggests the following interpretation. A smaller fraction of stratiform precipitation allows for a higher moisture buildup during the nonprecipitating congestus stage and results in a larger afternoon deep convection peak. It is interesting to note that, similar to the ocean case, the amplitude of precipitation in the morning is not very sensitive to the variation of the stratiform rain fraction. We conclude by noting that lower stratiform fractions are perhaps more appropriate for the land regime since they tend to produce higher afternoon precipitation peaks. 
Similarly, sensitivity tests to variations in the congestus rain fraction $f_{c}$ are depicted in the bottom panel of Fig. 6. As mentioned above, nonprecipitating congestus is one of the key parameters that directly control the afternoon precipitation peak associated with the land regime. A small congestus precipitation fraction allows the afternoon congestus clouds to rain from 1200 to 1530 LST without exhausting all of the moisture stored during the morning, which nonetheless permits a secondafternoon peak in deep convective precipitation that is roughly similar to the standard case corresponding to $f_{c}=0$ but somewhat smaller in magnitude. The high $f_{c}=0.2$, on the other hand, induces a significant drying of the troposphere by congestus rain and results in a weak (nonexisting in this extreme case) afternoon deep convection. Even though we have a high afternoon precipitation peak due to the high congestus rain fraction in the continental regime, this solution is unphysical since, as mentioned above, the bulk of the tropical land precipitation comes from deep convection and the trailing stratiform clouds (Casey et al. 2007; Takayabu et al. 2010).

\section{Concluding summary and discussion}

The multicloud model (KM06; KM08) with full bulk boundary layer dynamics (WK09) is used here to study the diurnal cycle of tropical precipitation over land. The case of precipitation over the ocean is presented in Part I. The main model consists of the two first vertical baroclinic modes of vertical structure forced by heating profiles based on the three cloud types that characterize organized tropical convection: cumulus congestus, deep convection, and trailing stratiform cloud decks, coupled to a thin boundary layer that responds to solar surface heating and downdrafts. This particular iteration of the model, introduced in WK09, incorporates the bulk-boundary layer equations of Stevens (2006) to facilitate the treatment of sensible and latent heat fluxes separately and includes entrainment and detrainment fluxes associated with shallow cumulus. Additional features of the present setup to accommodate tropical convection over land include a strong surface sensible heat flux (a large Bowen ratio), a strong temperature inversion, and nonzero entrainment and downdraft fluxes of sensible heat. The main result here is a stable periodic solution characterized by a large afternoon precipitation peak resembling observations of the diurnal cycle over tropical rain forests (e.g., Amazonia) (Machado et al. 2004).

The equations of motion along the equator-without rotation and without meridional dependence, forced by latent and sensible heat surface fluxes-are written in a moving frame circling the globe at a constant speed of one rotation per day following the local solar time, to facilitate the analysis. Time-independent solutions depicting the diurnal variations of the climatology where the effects of waves and other nonhomogeneities are filtered out are sought in the form of stable periodic solution of an ODE system with periodic coefficients.

The present study highlights the importance of capturing interactions of the free troposphere with the boundary layer, which is directly forced by the diurnal cycle of solar heating in terms of both sensible and latent heat fluxes. Since the model allows for efficient coupling between the boundary layer and free tropospheric potential temperature, the surface sensible heat flux warms the atmosphere in the morning, lowering the relative humidity and impeding deep convection. Boundary layer moisture, which peaks in the afternoon, combined with detrainment of shallow cumulus and nonprecipitating cumulus congestus clouds rapidly moistens the previously warmed troposphere. This then results in a pronounced afternoon precipitation maximum. According to the present model results, the detailed diurnal cycle of precipitation over land is divided into a cycle of five phases. 1) A persistent phase of overnight RCE level precipitation where convective heating balances the imposed longwave radiations prevails roughly between 2000 and 0600 LST. This is followed by 2) a CAPE buildup phase associated with the early morning warming of the boundary layer, which expands between 0600 and roughly 1000 LST, followed by a significant rise of precipitation that accelerates the drying and contributes to the warming of the middle troposphere; these effects in addition to the indirect warming due to mixing from shallow cumulus entrainment overcome the imposed radiative cooling. 3) A rapid and intense (re)moistening phase then starts at 1000 LST and is accompanied by congestus heating. 4) An explosive precipitation peak phase starts at 1600 LST due to the large amount of CAPE and moisture stored in the previously warmed atmosphere. It quickly dries the troposphere and consumes CAPE by further warming the troposphere and dissipates slightly before 1800 LST. The latter is followed by 5) a second (re)moistening episode that (re)establishes the moisture level to sustain the overnight precipitation of phase 1 and closes the cycle.

In this climatological setup, where wave oscillations are ignored, horizontal velocity variations are very small (see Fig. 2) and thus can be neglected. This is exploited in section $3 \mathrm{~b}$ to derive a single-column model approximation that is particularly amenable to Floquet stability analysis, which in fact confirmed that all the one-day solutions reported here are Floquet stable, consistent with the 
numerical results that implicitly suggest that these solutions are nonlinearly stable. Moreover, the extreme quantitative and qualitative resemblance between the full model and single-column model solutions provides solid evidence that large-scale moisture convergence is of secondary importance for the model's response to the diurnal cycle of solar heating.

Sensitivity tests that determine the main physical parameters crucial for explaining the phase shift in the precipitation maximum in the continental regime from morning to afternoon, as compared to the ocean case reported in Part I, are conducted and presented in section 4 . The results in section 4 demonstrate that the manifestation of an afternoon peak in deep convection is controlled by the combined effects of 1) a large Bowen ratio (a relatively large surface flux of sensible heat) of about $\beta=0.4,2$ ) a strong temperature inversion at RCE of about $-7.5 \mathrm{~K}, 3$ ) significant mixing of sensible heat at the top of the ABL due to entrainment and downdraft fluxes $\left(\alpha_{E}=\beta_{D}=1 / 3\right)$, and 4) weakly or nonprecipitating congestus heating $\left(f_{c}=0\right)$ to allow significant moisture buildup at noon and during early afternoon.

Although simple, the present model allows us to derive a physically sound five-phase cycle of tropical precipitation over land that is characterized by a strong afternoon precipitation maximum consistent with observations (Machado et al. 2004) and to suggest a minimal set of physical parameters that are crucial for its occurrence in parameterized model.

Acknowledgments. The research of BK is supported by a grant from the Natural Sciences and Engineering Research Council of Canada and the Canadian Foundation for Climate and Atmospheric Sciences. The research of AJM is supported by the National Science Foundation (NSF) Grant DMS-0456713, the office of Naval Research (NR) Grant N00014-05-1-0164, and the Defense Advanced Projects Agency Grant N0014-07-10750. YF is a postdoctoral fellow supported through AJM's above NSF and ONR grants. This research was partly performed when BK was visiting the Courant Institute during his sabbatical year, 2009-10, and was completed at the Institute for Pure and Applied Mathematics when both YF and BK were taking part as long-term visitors in the context of the long program on Climate and Data Hierarchies and AJM was a main organizer and participant.

\section{REFERENCES}

Casey, S. P. F., A. E. Dessler, and C. Schumacher, 2007: Frequency of tropical precipitating clouds as observed by the Tropical
Rainfall Measuring Mission Precipitation Radar and ICESat/ Geoscience Laser Altimeter System. J. Geophys. Res., 112, D14215, doi:10.1029/2007JD008468.

Chapin, F. S., P. Matson, and H. Mooney, 2002: Principles of Terrestrial Ecosystem Ecology. Springer, 436 pp.

Dai, A., and K. E. Trenberth, 2004: The diurnal cycle and its depiction in the Community Climate System Model. J. Climate, 17, 930-951.

Fisch, G., J. Tota, L. A. T. Machado, M. A. F. Silva Dias, R. F. da F. Lyra, C. A. Nobre, A. J. Dolman, and J. H. C. Gash, 2004: The convective boundary layer over pasture and forest in Amazonia. Theor. Appl. Climatol., 78, 47-59.

Frenkel, Y., B. Khouider, and A. J. Majda, 2011: Simple multicloud models for the diurnal cycle of tropical precipitation. Part I: Formulation and the case of the tropical oceans. J. Atmos. Sci., 68, 2169-2190.

Fu, R., B. Zhu, and R. E. Dickinson, 1999: How do atmosphere and land surface influence seasonal changes of convection in the tropical Amazon? J. Climate, 12, 1306-1321.

Hsu, S. A., 1998: A relationship between the Bowen ratio and seaair temperature difference under unstable conditions at sea. J. Phys. Oceanogr., 28, 2222-2226.

Johnson, R. H., T. M. Rickenbach, S. A. Rutledge, P. E. Ciesielski, and W. H. Schubert, 1999: Trimodal characteristics of tropical convection. J. Climate, 12, 2397-2418.

Khouider, B., and A. J. Majda, 2006: A simple multicloud parameterization for convectively coupled tropical waves. Part I: Linear analysis. J. Atmos. Sci., 63, 1308-1323.

—_, and _ 2007: A simple multicloud parameterization for convectively coupled tropical waves. Part II: Nonlinear simulations. J. Atmos. Sci., 64, 381-400.

$\ldots$, and _ 2008a: Equatorial convectively coupled waves in a simple multicloud model. J. Atmos. Sci., 65, 3376-3397.

$\longrightarrow$, and — 2008b: Multicloud models for organized tropical convection: Enhanced congestus heating. J. Atmos. Sci., 65, 895-914.

—, A. St-Cyr, A. J. Majda, and J. Tribbia, 2011: The MJO and convectively coupled waves in a coarse-resolution GCM with a simple multicloud parameterization. J. Atmos. Sci., 68, 240-264.

Kikuchi, K., and B. Wang, 2008: Diurnal precipitation regimes in the global tropics. J. Climate, 21, 2680-2696.

Lin, X., D. A. Randall, and L. D. Fowler, 2000: Diurnal variability of the hydrologic cycle and radiative fluxes: Comparisons between observations and a GCM. J. Climate, 13, 4159-4179.

Machado, L. A. T., H. Laurent, N. Dessay, and I. Miranda, 2004: Seasonal and diurnal variability of convection over the Amazonia: A comparison of different vegetation types and large scale forcing. Theor. Appl. Climatol., 78, 61-77.

Randall, D. A., Harshvardhan, and D. A. Dazlich, 1991: Diurnal variability of the hydrological cycle in a general circulation model. J. Atmos. Sci., 48, 40-62.

Raymond, D. J., 1995: Regulation of moist convection over the west Pacific warm pool. J. Atmos. Sci., 52, 3945-3959.

Sadhuram, Y., T. V. Ramana Murthy, Y. V. B. Sarma, and V. S. N. Murty, 2001: Comments on "On the estimation of overwater Bowen ratio from sea-air temperature difference." J. Phys. Oceanogr., 31, 1933-1934.

Schumacher, C., and R. Houze, 2003: Stratiform rain in the tropics as seen by the TRMM precipitation radar. J. Climate, 16, 17391756. 
Stevens, B., 2006: Bulk boundary-layer concepts for simplified models of tropical dynamics. Theor. Comput. Fluid Dyn., 20, 279-304.

Stull, R., 1988: An Introduction to Boundary Layer Meteorology. Springer, $380 \mathrm{pp}$.

Takayabu, Y. N., S. Shige, W.-K. Tao, and N. Hirota, 2010: Shallow and deep latent heating modes over tropical oceans observed with TRMM PR spectral latent heating data. J. Climate, 23, 2030-2046.

Tian, B., B. J. Soden, and X. Wu, 2004: Diurnal cycle of convection, clouds, and water vapor in the tropical upper troposphere:
Satellites versus a general circulation model. J. Geophys. Res. Lett., 109, D10101, doi:10.1029/2003JD004117.

Waite, M. L., and B. Khouider, 2009: Boundary layer dynamics in a simple model for convectively coupled gravity waves. J. Atmos. Sci., 66, 2780-2795.

Yang, G., and J. Slingo, 2001: The diurnal cycle in the tropics. Mon. Wea. Rev., 129, 784-801.

Yang, S., and E. A. Smith, 2006: Mechanisms for diurnal variability of global tropical rainfall observed from TRMM. J. Climate, 19, 5190-5226. 